\title{
Treatment Development for Alzheimer's Disease: How Are We Doing?
}

\author{
Constantin George Lyketsos
}

Over 40 million people worldwide suffer from dementia. This number is projected to exceed 110 million by 2050 because of the aging of the worldwide population, especially in lower- and middle-income countries. The most common cause of dementia is believed to be Alzheimer's, a brain disease associated with deposition of beta-amyloid protein and hyperphosphorylation of intraneuronal tau protein leading to synaptic degradation, neuronal loss, brain circuit disruption, a range of symptoms, and eventually, if the person lives long enough, death. Over the last few decades, treatment development has focused on the deposition of beta-amyloid protein (A-beta 1-42) that is produced in the brain in huge quantities continuously and is thought to be toxic. Unfortunately, amyloid oriented therapies targeting individuals with dementia, or its prodrome mild cognitive impairment (MCI), have not been successful therapeutically even though they have been associated with reductions in amyloid. Currently, efforts are underway to deliver these therapies to individuals with very early symptoms or at risk for Alzheimer's dementia by virtue of genetics or a brain amyloid PET scan. Results from these studies are expected to begin to emerge by early 2020. In the meantime, since the amyloid hypothesis has been called into question, a number of different avenues are being pursued for treatment development. These are driven in part by new findings related to the polygenic nature of Alzheimer's as well as the interaction between this brain disease with factors such as brain vascular disease, insulin resistance, and/or brain inflammation. The expected future of $\mathrm{AD}$ treatment development is thought to be precision medicine.

C. G. Lyketsos $(\bowtie)$

Johns Hopkins University, Baltimore, MD, USA

e-mail: kostas@jhmi.edu 